Meta

Journal des traducteurs

Translators' Journal

\title{
Ostbelgien spricht viele Sprachen
}

\section{Guido Thomé}

Volume 39, numéro 1, mars 1994

La traduction et l'interprétation dans la Belgique multilingue

URI : https://id.erudit.org/iderudit/004489ar

DOI : https://doi.org/10.7202/004489ar

Aller au sommaire du numéro

\section{Éditeur(s)}

Les Presses de l'Université de Montréal

ISSN

0026-0452 (imprimé)

1492-1421 (numérique)

Découvrir la revue

Citer cet article

Thomé, G. (1994). Ostbelgien spricht viele Sprachen. Meta, 39(1), 95-96. https://doi.org/10.7202/004489ar

\section{Résumé de l'article}

On ne peut s'étonner que dans un pays qui compte à peine 10 millions d'habitants et possède trois langues officielles, que des difficultés de communication soit préprogrammées. Si les querelles linguistiques sont souvent en témoins de ces incompréhensions, la troisième langue n'est pas souvent mentionnée. Les habitants de cette petite communauté ont fait « de nécessité loi » et se sont zieutés vers le plurilinguisme. La Belgique germanophone n'est cependant pas « l'eldorado » de la traduction. Les droits de 70000 habitants sont à revendiquer dans la langue de la majorité. Le Belge de l'Est en polyglotte est le mot d'ordre pour la survie et " qualité », seule solution pour faire face à la dégradation.
Tous droits réservés @ Les Presses de l'Université de Montréal, 1994
Ce document est protégé par la loi sur le droit d'auteur. L'utilisation des services d'Érudit (y compris la reproduction) est assujettie à sa politique d'utilisation que vous pouvez consulter en ligne.

https://apropos.erudit.org/fr/usagers/politique-dutilisation/ 


\title{
OSTBELGIEN SPRICHT VIELE SPRACHEN
}

\author{
GUIDO THOME
}

\begin{abstract}
Résumé
On ne peut s'étonner que dans un pays qui compte à peine 10 millions d'habitants et possède trois langues officielles, que des difficultés de communication soit préprogrammées. Si les querelles linguistiques sont souvent en témoins de ces incompréhensions, la troisième langue n'est pas souvent mentionnée. Les habitants de cette petite communauté ont fait «de nécessité loi» et se sont zieutés vers le plurilinguisme. La Belgique germanophone n'est cependant pas «l' eldorado» de la traduction. Les droits de 70000 habitants sont à revendiquer dans la langue de la majorité. Le Belge de l'Est en polyglotte est le mot d'ordre pour la survie et «qualité», seule solution pour faire face à la dégradation.
\end{abstract}

In einem Land, das drei Landessprachen sein eigen nennt, und das bei knapp zehn Millionen Einwohnern, sind Verständigungsschwierigkeiten vorprogrammiert. Der belgische Sprachenstreit, der dem kleinen Königreich oft Schlagzeilen im Ausland besorgt(e), ist zu einem illustren Zeugen dieser Schwierigkeiten geworden. Doch bezeichnenderweise ist bei diesem Sprachenstreit nur selten von der kleinsten Sprachgemeinschaft des Landes die Rede, vom deutschsprachigen Teil Belgiens. Bezeichnend ist dies für die Bevölkerung dieses Landstriches mit wechselvoller Geschichte, die - um es mit einem deutschen Sprichwort zu sagen - aus der Not eine Tugend gemacht hat. Eine Tugend, die aus den Ostbelgiern gesuchte Objekte auf dem belgischen Arbeitsmarkt macht ihrer Mehrsprachigkeit wegen. Übersetzen gehört in Ostbelgien zum Alltag wie die Butter zum Brot. Wer am Rande von Sprachengrenzen wohnt und zu einer Minderheit innerhalb eines kleinen Landes gehört, kann es sich nicht leisten, sich auf den Lorbeeren seiner Muttersprachenkenntnisse auszuruhen.

Ostbelgien - ein Dorado für Übersetzer also? Mitnichten! Dem Verfasser dieser Zeilen sind zwar einige Übersetzer ostbelgischer Herkunft bekannt, die im Inland ihr Geld verdienen, doch das Land dies- und jenseits des Hohen Venns scheint noch brach zu liegen, was Übersetzungen betrifft.

Hinter dem ostbelgischen Übersetzer-Dilemma steckt die Tücke des Alltags. Wenn 70.000 Menschen in einem Land mit zehn Millionen Bevölkerung ihr Recht fordern, müssen sie dies - auch - in der Sprache der Mehrheit tun, wollen sie nicht als Exoten hinter Zoo-Gitterstäben verkümmern. Deshalb oder besser: auch deshalb hat der Ostbelgier aus der Not, Sprachen zu lemen, um sich in seinem eigenen Land verständlich machen zu können, eine Tugend gemacht, die Tugend der Mehrsprachigkeit. So weit so gut.

Das Dilemma trägt den Namen Qualität. Und verschlimmert sich mit der zahl derer, die sich berufen fühlen. Übersetzungen sind in Ostbelgien zu etwas Alltäglichem geworden. Angefangen hat auf offizieller Seite alles mit dem Erscheinen des ersten Staatsblattes mit deutschen Texten im Jahre 1920. Seitdem hat die Übersetzerkunst in Ostbelgien seltene Blüten der deutschen Amtssprache getrieben, die sich allerdings - auf Dauer - durchsetzen konnten, weil keiner da war, der ihnen den Garaus gemacht hätte. Offizielle Stellen mühen sich inzwischen zwar redlich, doch für den ostbelgischen Otto Normalverbraucher sind Briefe in deutscher Sprache aus dem belgischen Inland immer noch eine Quelle ... der Belustigung, wenn der Empfänger mit einer kräftigen Prise Humor den Schriftverkehr mit seiner Versicherungsgesellschaft - um ein Beispiel von vielen möglichen zu zitieren. Doch das Lachen ist einem schnell vergangen, wenn Phantasiereichtum und bester Wille 
nicht ausreichen, den Inhalt dieser offiziellen Schreiben zu erahnen. Nun mag man mit viel Wohlwollen die Briefeschreiber aus dem belgischen Inland in Ostbelgien noch entschuldigen und ihnen für ihre Bemuihungen danken.

Unverständlicher ist in meinen Augen vor allem die Gleichgültigkeit, mit der Werbemacher und Hersteller, die für IHR Produkt werben möchten und dies auch tun, die Sprache als Träger ihrer Mitteilungen völlig ignorieren. Oder besser gesagt: auf sie keinen Wert legen. Darf man daraus den Schluß ziehen, daß es in Ostbelgien um Übersetzungen nicht gut bestellt ist, weil es um die Sprache - in diesem Fall die deutsche - nicht gut bestellt ist? Ich fürchte ja! Vielen Ostbelgiern darf man bescheinigen, daß sie sich mit allen Belgiern - ob Flamen oder Wallonen - in deren Muttersprache unterhalten können. Doch vielen Ostbelgiern muß man leider auch bescheinigen, daß Sie sich in keiner - leider auch nicht in ihrer Muttersprache Deutsch - Sprache besonders gut ausdruicken können. Nun sollte man daraus nicht schließen, daß wir in Ostbelgien uns mit Händen und Füßen verständigen, doch großen Teilen der Bevölkerung mangelt es an Wortschatz, an elementaren Kenntnissen der Grammatik und nicht zuletzt an Sprachgefühl. Deshalb wird dieser Mangelzustand auch nicht als solcher und damit als verbesserungsbedürftig empfunden. Zum > Überleben< reichen die Kenntnisse allemal aus. Und daß deutsche Nachbarn uns manchmal befremdet anblicken, haben wir längst mit der Gewißheit akzeptiert, daß Bayern und Berliner schließlich auch nicht das reinste Hochdeutsch reden. Doch mit dem feinen Unterschied, daß diese Hochdeutsch sehr wohl noch von ihrem eigenen Dialekt unterscheiden können. Und das ihre Mundart Charme besitzt und >reich< ist. In Ostbelgien wird an manchen Stellen sogar der Verlust des Plattdeutschen beweint. Dem wäre auch ungeteilt zuzustimmen, wenn sich dieses Plattdeutsche nicht schon lange zu einer armen Sprache entwickelt hätte, die mit einem ca. 400 Worten umfassenden Aktivwortschatz bei einer Diskussion über etwas anderes als Wetter und Fußball sogleich auf hochdeutsche oder fremdsprachige Hilfe angewiesen ist. Abgesehen von wenigen Ausnahmen ist der Dialekt in Ostbelgien nicht sprachbereichernd.

Ein weiteres Indiz für den schwierigen Stand, den die deutsche Sprache in Ostbelgien hat, ist die geringe literarische Produktivität. Nun wäre es sicher vermessen, von einer ca. 70.000 Personen umfassenden Bevölkerungsgruppe zu erwarten, sie bringe Literaturnobelpreisträger hervor, doch viel mehr als die zaghaften Versuche eines Freddy Derwahl und der Literaturzeitschrift $>$ Krautgarten $<$ kann Ostbelgien an zeitgenössischer Literatur in deutscher Sprache nicht vorzeigen.

Wie soll in einer Gesellschaft, in der die Sprache nicht — so sehr — geachtet wird, wie es sich die, die sich mit ihr beschäftigen, wünschen würden, wie soll in solch einer Gesellschaft ein fruchtbarer Boden für GUTE Übersetzungen entwickeln? Unmöglich. Denn mit derselben Selbstverständlichkeit, mit der die Muttersprache nicht gepflegt wird, fühlen sich plötzlich Tausende zum Übersetzen berufen. Denn Übersetzungen werden sehr wohl gebraucht. Allerdings für den - oft - einmaligen und - meist - internen (sprich: innerostbelgischen) Gebrauch. Allzu deutlich wird dieser Mißstand wieder auf dem Gebiet der Werbung, die übersetzt zumeist in geschriebener Form in der einzigen ostbelgischen Tageszeitung - dem Grenz-Echo - und in einigen Werbeblättern oder auf Plakaten auf die Bevölkerung losgelassen wird. Doch ist deshalb die Nonchalance zu verzeihen, mit der Agenturen nationale Werbung in Ostbelgien verkaufen oder mit der regionale Anbieter ihre Produkte an den Mann zu bringen versuchen? Ich denke nicht, aber so lange niemand protestiert, wenn - wie geschehen - ein Anbieter von Elektrowaren vor Weihnachten Prospekte in alle Haushalte verschickt, in denen es vor Fehlern nur so wimmelt, wenn ein französischer Automobilhersteller seinen Kunden mitteilt, sie müßten bekloppt (sic) sein, wenn sie sich jetzt nicht für sein Modell X entscheiden. Da ist es kein Wunder, wenn sich kein Übersetzerbüro in Ostbelgien etabliert, bzw. etablieren kann, schließlich hat auch noch niemand versucht, in Sibirien einen Vergnügungspark zu eröffnen. 